\title{
Sentinel lymph node biopsy in elderly breast cancer
}

\author{
S Ricciardi ${ }^{*}$, C Terranova ${ }^{2}$, R Gianesini ${ }^{1}$, E Mion ${ }^{1}$, F Viano ${ }^{1}$, L Faccin ${ }^{1}$, L De Santis ${ }^{1}$ \\ From de Senectute: Age and Health Forum \\ Catanzaro, Italy. 5-7 December 2009
}

\section{Background}

Elderly patients make up a large part of the breast cancer population and there are important specific considerations for this population. Studies of elderly women have found that Breast conservation therapy (ie, lumpectomy, axillary lymph node sampling, and postoperative RT) is often associated with better quality of life. Our Purpose was to evaluate the performance and feasibility of sentinel lymph node biopsy in patients aged $\geq 65$ in Day surgery (DS).

\section{Materials and methods}

Between January 2005 and July 2009, we performed 53 Sentinel Node Biopsy (SNB), in patients with median age of 66 years, (range 24-87) with tumors of less than 3 $\mathrm{cm}$ with clinically negative axilla.

The sentinel node was successfully identified in all the cases $(100 \%)$ using technetium-99m $\left({ }^{99 \mathrm{~m}} \mathrm{Tc}\right)$ sulphur colloid, injected the day before surgery. The following day the surgeon identified the sentinel node by gamma probe and marked it on the skin. We never used vital blue dye. In the great majority of patients we performed SNB and quadrantectomy in day surgery (DS) and local anaesthesia (LA) using the same incision or through a separate small incision in the axilla $(2-3 \mathrm{~cm})$. This approach was discussed with all patients and informed consent was obtained. The whole procedure took a median time of 40 minutes and all the patients tolerated the procedure very well with minimum discomfort.

\section{Results}

We identified the SLN in all patients (100\%). The most common histological group was ductal carcinoma. Complete standard axillary dissection followed only if the sentinel node contained metastases. In 6 out of the
18 cases (32\%) with istologically node-positive breast cancer, the sentinel node was the only lymph node affected. 2 cases showed a double SLN in the axilla. In the group with a metastatic node $5(27 \%)$ were aged $\geq 65$ years. No axillary recurrences were observed. Our results indicate that no significant disparities exist in the sampling of axillary lymph nodes among women based solely on their age.

\section{Conclusion}

This procedure is safe and well accepted in women aged 65 years and older. As regards hospital logistics, operations in DS and LA can be easily managed, leading to a significant cost reduction.

No significant disparities exist in the sampling of axillary lymph nodes among elderly women compared with younger women, and the whole procedure can be done in Day Surgery. The sentinel node biopsy remains a key component in determining stage, and thereby prognosis and appropriate treatment options also in elderly women.

\section{Author details}

${ }^{1}$ Chirurgia Generale, Ospedale "S. Lorenzo" Valdagno, VI, Italy. ${ }^{2}$ Department of Environmental Medicine, Hospital-University of Padova, Italy.

Published: 19 May 2010

doi:10.1186/1471-2318-10-S1-A29

Cite this article as: Ricciardi et al: Sentinel lymph node biopsy in elderly breast cancer. BMC Geriatrics 2010 10(Suppl 1):A29.

${ }^{1}$ Chirurgia Generale, Ospedale "S. Lorenzo" Valdagno, VI, Italy 